Pacific Journal of Mathematic 


\title{
LOCALIZATION AND SPLITTING IN HEREDITARY NOETHERIAN PRIME RINGS
}

\author{
K. R. GOODEARL
}

The purpose of this paper is to introduce a localization corresponding to any collection $X$ of maximal right ideals in an hereditary noetherian prime ring $R$. The localized ring $R_{X}$ has only as many simple right modules (up to isomorphism) as $R$ has simple right modules of the form $R / M$, where $M \in X$. In particular, for a single maximal right ideal $M$ the ring $R_{M}$ has exactly one simple right module (up to isomorphism). These localizations satisfy a globalization property in that a sequence of $R$-homomorphisms is exact if and only if it is exact when localized at each maximal right ideal of $R$. These localizations are also the most general possible, for it is shown that every ring. between $R$ and its maximal quotient ring has the form $R_{X}$ for suitable $X$. The relationship between these localizations and other previously introduced localizations for hereditary noetherian prime rings is discussed, and then this localization technique is applied to the question of when an hereditary noetherian prime ring $R$ can be a splitting ring (i.e., a ring such that the singular submodule of every right module is a direct summand). Such a ring is shown to be an iterated idealizer from a ring over which all singular right modules are injective. Finally, hereditary noetherian prime splitting rings are characterized by the properties of possessing a minimal two-sided ideal and having all faithful simple right modules injective.

All rings in this paper are associative with unit, and all modules are unital. We use the abbreviation HNP ring to stand for a ring $R$ which is right and left hereditary, right and left noetherian, and prime. We shall need a number of standard properties of an $H N P$ ring $R$, which we now list for reference. It follows from [18, Theorem 4] that every cyclic singular $R$-module is artinian, and in particular every proper factor ring of $R$ is right and left artinian. It also follows that every cyclic singular $R$-module has a composition series, and consequently every singular $R$-module has essential socle. We note from [5, Lemma 1.1] that the module $R_{R}$ has nonzero socle if and only if $R$ is simple artinian. According to [5, Theorem 2.1], all finitely generated nonsingular right $R$-modules are projective; in particular, this holds for finitely generated $R$-submodules of the maximal quotient ring of $R$. As a consequence of this result, we note that all nonsingular right $R$-modules must be flat.

Our notation for singular submodules coincides with that used 
in [8]: $\mathscr{S}(R)$ stands for the set of essential right ideals of $R, Z(A)$ or $Z_{R}(A)$ stands for the singular submodule of an $R$-module $A$, and $S^{0} R$ stands for the maximal right quotient ring of $R$. Also, we use $E(A)$ to denote the injective hull of a module $A$.

2. Localization. Given any collection $X$ of maximal right ideals of an $H N P$ ring $R$, we define $\mathscr{S}_{X}$ to be the set of those essential right ideals $I$ of $R$ for which $R / I$ has no composition factors isomorphic to any of the modules $R / M$, where $M \in X$. [We do allow the possibility that $X$ is empty, in which case $\mathscr{S}_{X}=\mathscr{S}(R)$.] We check that $\mathscr{S}_{X}$ satisfies the right-ideal analogues of [7, Theorems 2.1, 2.5], hence we obtain an idempotent kernel functor $T_{X}$ (i.e., a torsion theory) defined as follows:

$$
T_{X}(A)=\left\{x \in A \mid x I=0 \text { for some } I \in \mathscr{S}_{X}\right\} .
$$

Note that since $\mathscr{S}_{x} \subseteq \mathscr{S}(R)$, all nonsingular right $R$-modules are $T_{X}$-torsion-free.

To each right $R$-module $A$ is associated a module of quotients with respect to $T_{X}[7, \S 3]$, and we shall denote this module by $A_{X}$. The assignment of $A_{X}$ to $A$ is the object map of the localization functor associated with $T_{X}$, which we refer to as localization at $X$. Since $R_{R}$ is $T_{X}$-torsion-free, the ring of quotients $R_{X}$ is a quotient ring of $R$ and so may be identified with a subring of $S^{0} R$ :

$$
R_{X}=\left\{x \in S^{0} R \mid x I \subseteq R \text { for some } I \in \mathscr{S}_{X}\right\} \text {. }
$$

Proposition 1. Let $R$ be an HNP ring, $X$ any collection of maximal right ideals of $R$.

(a) Every right $R_{X}$-module is $T_{X}$-torsion-free (as an $R$-module).

(b) $I R_{X}=R_{X}$ for every $I \in \mathscr{S}_{X}$.

(c) Localization at $X$ is naturally equivalent to the functor $(-) \bigotimes_{R} R_{X}$.

Proof. According to [7, Theorem 4.3], it suffices to show that localization at $X$ is right exact and commutes with direct sums. These properties follow from [7, Theorems 4.4,4.5] because $R$ is right noetherian and right hereditary.

We need to know that the ring $R_{X}$ is again an HNP ring. This is in fact true of any ring between $R$ and $S^{0} R$, as the next proposition shows.

Proposition 2. Let $R$ be an HNP ring, and let $T$ be any subring of $S^{0} R$ which contains $R$. 
(a) $T$ is an HNP ring with maximal quotient ring $S^{0} R$.

(b) ${ }_{R} T$ and $T_{R}$ are both flat, and the natural map $T \otimes_{R} T \rightarrow T$ is an isomorphism.

( c) For any right (or left) T-modules $A$ and $B, \operatorname{Hom}_{T}(A, B)=$ $\mathrm{Hom}_{R}(A, B)$.

(d) For any right $T$-module $A$, the natural map $A \otimes_{R} T \rightarrow A$ is an isomorphism, and similarly for left T-modules.

(e ) $Z_{T}(A)=Z_{R}(A)$ for any $T$-module $A$.

Proof. (a) Clearly $S^{0} R$ is the maximal right and left quotient ring of $T$, whence $T$ is right and left finite-dimensional. Since [10, Proposition 1.6] shows that $R$ is right and left hereditary, [16, Corollary 2 to Theorem 2.1] says that $R$ is also right and left noetherian. Finally, since $R$ is a prime ring which is essential in $T$, we infer that $T$ must be prime.

(b) is [10, Proposition 1.5].

(c) and (d) follow from (b) by [17, Corollary 1.3].

(e) follows from (a) and (c) and the fact that over either ring, $Z(A)$ is the intersection of the kernels of all homomorphisms from $A$ into the maximal quotient ring $S^{0} R$. (See [8, Proposition 1.18].)

The usefulness of localization at $X$ is due to the fact that the ring $R_{X}$ has only as many isomorphism classes of simple right modules as there are isomorphism classes of simple right $R$-modules of the form $R / M$, where $M \in X$. This is the content of the following theorem. In particular, when $X$ contains exactly one maximal right ideal $M$, it follows that $R_{X}$ has exactly one simple right module (up to isomorphism). In this case, we write $R_{M}$ for $R_{\{M\}}$, etc.

THEOREM 3. Let $R$ be an HNP ring, $X$ any nonempty collection of maximal right ideals of $R$.

(a) For any $M \in X, M R_{X}$ is a maximal right ideal of $R_{X}$ and the natural map $f: R / M \rightarrow R_{X} / M R_{X}$ is an essential monomorphism.

(b) For any $M, N \in X, R_{X} / M R_{X} \cong R_{X} / N R_{X}$ if and only if $R / M \cong R / N$.

(c) Any simple right $R_{X}$-module $A$ is isomorphic to $R_{X} / M R_{X}$ for some $M \in X$.

Proof. (a ) Observing that $M R_{X} / M$ is $T_{X}$-torsion while $R / M$ is $T_{X}$-torsion-free, we see that $(R / M) \cap\left(M R_{X} / M\right)=0$, from which it follows that $f$ is a monomorphism. Given any $x \in R_{X}$ such that $x \notin M R_{X}$, we have $x J \subseteq R$ for some $J \in \mathscr{S}_{X}$. Inasmuch as $J R_{X}=R_{X}$ by Proposition 1 , we see that $x J \nsubseteq M$ and so $x R \cap R \nsubseteq M$. Therefore, $f(R / M)$ is essential in $R_{X} / M R_{X}$. 
Now $f(R / M)$ is a simple, essential $R$-submodule of $R_{X} / M R_{X}$ and thus is contained in every nonzero $R$-submodule of $R_{X} / M R_{X}$. It follows easily that $R_{X} / M R_{X}$ is simple as an $R_{X}$-module.

(b) If $R / M \cong R / N$, then clearly $R_{X} / M R_{X} \cong R_{X} / N R_{X}$. The converse follows from the fact that $R_{X} / M R_{X}\left(R_{X} / N R_{X}\right)$ has a unique simple $R$-submodule, which is isomorphic to $R / M(R / N)$.

(c) If $R_{X}$ is simple artinian, then all simple right $R_{X}$-modules are isomorphic and we are done because $X$ is nonempty. Otherwise $R_{X}$ has zero right socle, whence the simple right module $A$ must be singular. According to Proposition 2, $A$ is also singular as an $R$-module, whence $A$ must contain a simple $R$-submodule. Inasmuch as $A$ is $T_{X}$-torsion-free by Proposition 1 , this simple $R$-submodule must be isomorphic to $R / M$ for some $M \in X$. Utilizing Proposition 2 again, we obtain a monomorphism $R_{X} / M R_{X} \rightarrow A \otimes_{R} R_{X} \rightarrow A$, and this map must be an isomorphism.

THEOREM 4. Let $R$ be an HNP ring, and let $E: A \stackrel{f}{\rightarrow} B \stackrel{g}{\rightarrow} C$ be any sequence of right $R$-homomorphisms. Then $E$ is exact if and only if the localized sequence $E_{M}: A_{M} \stackrel{f_{M}}{\rightarrow} B_{M} \stackrel{g_{M}}{\rightarrow} C_{M}$ is exact for every maximal right ideal $M$ of $R$.

Proof. The localization $E_{M}$ is just the tensor product of $E$ with the flat left $R$-module $R_{M}$, hence if $E$ is exact it follows that every $E_{M}$ must be exact.

Now assume conversely that every $E_{M}$ is exact.

Case I. $\quad C=0$.

The exactness of $E_{M}$ says that each $f_{M}$ is an epimorphism, i.e., $B_{M}=(f A)_{M}$. Thus $(B / f A)_{M}=0$ for each $M$, whence $B / f A$ is $T_{M^{-}}$ torsion for every $M$. Given any $x \in B / f A$, it now follows that $x R$ has a composition series with no composition factors isomorphic to $R / M$ for any $M$. Therefore, $B / f A=0$, i.e., $f$ is an epimorphism.

\section{Case II. General case.}

Since $g_{M} f_{M}=0$ for all $M,(g f A)_{M}=0$ for all $M$, whence $g f A=0$ as in Case I. As in the commutative proof, we now obtain $[(\operatorname{ker} g) / f A]_{M}=0$ for all $M$, and thus $\operatorname{ker} g=f A$.

Inasmuch as Proposition 2 shows that all rings between an $H N P$ ring $R$ and its quotient ring $S^{\circ} R$ are again $H N P$ ring, the question arises whether all such intermediate rings are of the form $R_{X}$ for 
suitable $X$. The following theorem provides an affirmative answer to this question.

THEOREM 5. Let $R$ be an HNP ring which is not artinian, and let $W$ be a collection of maximal right ideals of $R$ such that each simple right $R$-module is isomorphic to $R / M$ for exactly one $M \in W$. Then the assignment $X \mapsto R_{X}$ gives a 1-1 order-reversing correspondence between the set $\mathscr{F}$ of subsets of $W$ and the set $\mathscr{K}$ of subrings of $S^{0} R$ which contain $R$.

Proof. For any $P \in \mathscr{K}$, let $\phi(P)$ denote the set of those $M \in W$ such that $R / M$ is not isomorphic to any composition factors of any submodule of $P / R$. The maps $\phi$ and $X \mapsto R_{X}$ are clearly orderreversing.

Given any $X \in \mathscr{F}$, it follows from the definition of $R_{X}$ that no composition factor of any submodule of $R_{X} / R$ can be isomorphic to $R / M$ for any $M \in X$. Therefore $X \subseteq \phi\left(R_{X}\right)$. Now consider any $K \in W$ which does not belong to $X$. Inasmuch as $R$ is not artinian, $\operatorname{soc}\left(R_{R}\right)=0$ and so $\operatorname{Ext}_{R}^{1}(R / K, K) \neq 0$. However, $K$ is a finitely generated projective right $R$-module, hence it follows that $\operatorname{Ext}_{R}^{1}(R / K, R) \neq 0$. Thus there exists a map $f: K \rightarrow R$ which does not extend to a map $R \rightarrow R$. Now $f$ must be left multiplication by some $u \in Q$ such that $u \notin R$, hence we obtain $(u R+R) / R \cong R / K$. Since $K \notin X,(u R+R) / R \neq R / M$ for all $M \in X$, and thus $u \in R_{X}$. But now $R / K$ is isomorphic to a submodule of $R_{X} / R$, whence $K \notin \phi\left(R_{X}\right)$. Therefore $X=\phi\left(R_{X}\right)$.

Given any $P \in \mathscr{K}$, it follows from the definition of $\phi(P)$ that no composition factor of any submodule of $P / R$ can be isomorphic to $R / M$ for any $M \in \phi(P)$, and thus $P \subseteq R_{\phi(P)}$. Conversely, we must show that any $x \in R_{\phi(P)}$ belongs to $P$, and we proceed by induction on the length $k$ of the module $(x R+R) / R$. If $k=0$, then $x \in R \subseteq P$, so now let $k>0$ and assume that $y \in P$ whenever $y \in R_{\phi(P)}$ and $(y R+R) / R$ has length less than $k$.

Choose a submodule $H / R$ of $(x R+R) / R$ with length $k-1$, and note from the induction hypothesis that $H \subseteq P$. Now $(x R+R) / H \cong$ $R / M$ for some $M \in W$, and since $x \in R_{\phi(P)}$ we must have $M \notin \phi(P)$. Thus there must exist a submodule of $P / R$ with a composition factor isomorphic to $R / M$, hence we can find finitely generated right $R$ modules $C, D$ with $R \cong C \cong D \subseteq P$ such that $D / C \cong R / M$. As observed in the introduction, $D$ is projective, hence the isomorphism $D / C \rightarrow$ $(x R+R) / H$ lifts to a map $f: D \rightarrow x R+R$ such that $f C \subseteq H$ and $f D+H=x R+R$. Now $f$ must be left multiplication by some $u \in S^{0} R$, and since $u R \subseteq f C \subseteq H \subseteq P$ we have $u \in P$. However, $D, H \subseteq P$ as well, and so $x R+R=u D+H \subseteq P$. Therefore, the induction 
works and we obtain $P=R_{\phi(P)}$.

For a given maximal right ideal $M$ of $R$, Theorem 3 shows that at least all simple right $R_{M}$-modules are isomorphic, but this is not enough to show that $R_{M}$ is local in any sense of the term. For example, if $R$ is the ring of differential polynomials constructed in [4, Theorem 1.4], then $R$ is a simple HNP ring such that all simple right $R$-modules are isomorphic. Then $R_{M}=R$ for any maximal right ideal $M$ of $R$, but $R$ modulo its Jacobson radical is not even artinian.

On the other hand, we can show that $R_{M}$ is local in a certain sense provided that $M$ contains a nonzero maximal two-sided ideal, and that $R$ is a Dedekind prime ring, i.e., an $H N P$ ring which is a maximal order in its quotient ring (or equivalently [6, Theorem 1.2] an $H N P$ ring with no nontrivial idempotent two-sided ideals). We proceed via the the following lemma, which is also needed later.

Lemma 6. Let $R$ be an HNP ring, $X$ any collection of maximal right ideals of $R$. Let $M \in X$, and let $f: R / M \rightarrow R_{X} / M R_{X}$ be the natural map. If $S$ is any simple $R$-submodule of $\left(R_{X} / M R_{X}\right) / f(R / M)$, then $S \not R / K$ for any $K \in X$.

Proof. Suppose on the contrary that $S \cong R / K$ for some $K \in X$, and let $A$ denote the submodule of $R_{X} / M R_{X}$ containing $f(R / M)$ such that $A / f(R / M)=S$. Then we have an exact sequence $0 \rightarrow R / M \rightarrow$ $A \rightarrow R / K \rightarrow 0$. Localizing this sequence at $X$ gives another exact sequence $0 \rightarrow R_{X} / M R_{X} \rightarrow A_{X} \rightarrow R_{X} / K R_{X} \rightarrow 0$, from which we infer that $A_{X}$ has length 2 as an $R_{X}$-module. However, we also have a monomorphism $A_{X} \rightarrow\left(R_{X} / M R_{X}\right)_{X}=R_{X} / M R_{X}$, which is absurd because $R_{X} / M R_{X}$ is simple.

Theorem 7. Assume that $R$ is a Dedekind prime ring. Let $P$ be a nonzero maximal two-sided ideal of $R$, and let $M$ be any maximal right ideal of $R$ which contains $P$. Then $P R_{M}$ is the Jacobson radical of $R_{M}$, and $R_{M} / P R_{M}$ is a simple artinian ring.

Proof. We first claim that the natural map $f: R / M \rightarrow R_{M} / M R_{M}$ is an isomorphism. If not, then Theorem 3(a) shows that $\left(R_{M} / M R_{M}\right) / f(R / M)$ is a nonzero singular module, hence $R_{M} / M R_{M}$ has a submodule $A$ containing $f(R / M)$ such that $A / f(R / M)$ is simple. Inasmuch as $f(R / M)$ is essential in $A, A$ must be indecomposable, whence [5, Theorem 3.9] says that $A$ is either completely faithful or else unfaithful. Now $f(R / M)$ is annihilated by $P$ and hence is unfaithful, so $A$ cannot be completely faithful. Thus $A$ is unfaithful, and 
consequently $A / f(R / M)$ must be unfaithful. Choosing a maximal right ideal $K$ such that $R / K \cong A / f(R / M)$, we thus see that $K$ is bounded, i.e., the two-sided ideal $H=\{r \in R \mid R r \subseteq K\}$ is nonzero. Now $R / H$ is an artinian primitive ring, hence simple. Inasmuch as $R / K \nsubseteq R / M$ by Lemma 6 , it follows that $H \nsubseteq P$. According to [6, Proposition 2.8], $H P=H \cap P$, whence $R / H P$ is a semisimple ring. Therefore $A$, which is an $(R / H P)$-module of length 2 , must be a direct sum of two simple modules, which contradicts the observation above that $A$ is indecomposable.

Thus $f$ is an isomorphism, whence $P$ is the annihilator in $R$ of $R_{M} / M R_{M}$. Inasmuch as all simple right $R_{M}$-modules are isomorphic to $R_{M} / M R_{M}$ by Theorem 3, the annihilator of $R_{M} / M R_{M}$ in $R_{M L}$ is the Jacobson radical $J$ of $R_{M}$, and we obtain $J \cap R=P$. We now have a monomorphism $R / P \rightarrow R_{M} / J$, which induces a monomorphism $R_{M} / P R_{M} \rightarrow$ $\left(R_{M} / J\right) \otimes_{R} R_{M} \rightarrow R_{M} / J$. Therefore $P R_{M}=J$. Since $J \neq 0$ and $R_{M}$ is an $H N P$ ring by Proposition 2, the factor ring $R_{M} / J$ must be artinian. Also, all simple right $R_{M}$-modules are isomorphic, and this forces $R_{M} / J$ to be a simple ring.

3. Relation to other localizations. In [1], K. Asano introduced a localization at a maximal two-sided ideal $P$ in a type of ring $R$ which is now known as a "bounded Asano order". Specifically, an Asano order (in a simple artinian ring $Q$ ) is a right and left noetherian prime ring $R$ which is an order in $Q$ such that the two-sided fractional $R$-ideals form a group, while a bounded Asano order is one in which every essential one-sided ideal contains a nonzero two-sided ideal. Asano and other later authors have shown that such a bounded Asano order $R$ is a right and left hereditary ring (the simplest proof is due to T. H. Lenagan in [13]). Thus $R$ is in particular an HNP ring.

The localized ring introduced by Asano, which we shall refer to as $A$ for the moment, consists of all elements $x$ in the quotient ring $S^{0} R$ such that $x I \cong R$ for some two-sided ideal $I$ of $R$ not contained in $P$. If $M$ is any maximal right ideal of $R$ which contains $P$, then it is easy to check that $A=R_{M}$ in the notation of the present paper. Since $R$ is clearly a Dedekind prime ring, the fact that $A$ is a local ring [1, Sätze 3.4,3.5] (in the sense used above) is now also a consequence of Theorem 7 .

In [10], J. Kuzmanovich introduced a localization at a maximal two-sided ideal $M$ in a Dedekind prime ring $R$, and later [11] generalized this to the situation where $R$ is an $H N P$ ring and $M$ is maximal among the invertible two-sided ideals of $R$. This localized ring $L$ (denoted $R_{M}$ in [10] and $Q_{f}$ in [11]) consists of all $x \in S^{0} R$ such that no composition factor of $(x R+R) / R$ is annihilated by $M$ 
[11, p. 149]. It is automatic from our definitions that $L=R_{X}$, where $X$ denotes the set of those maximal right ideals of $R$ which contain $M$. Kuzmanovich also defines an additional localization $S$ [10] (denoted $Q_{b}$ in [11]) consisting of those $x \in S^{0} R$ for which $(x R+R) / R$ is annihilated by an invertible two-sided ideal. It is easy to check that $S=R_{Y}$, where $Y$ denotes the set of those maximal right ideals of $R$ which do not contain an invertible two-sided ideal. Kuzmanovich shows that the collection of these localizations $L$, together with $S$, satisfy a globalization property analogous to Theorem 4 [10, Theorem 4.4 and Proposition 4.6], [11, Theorem 3.12]. These globalization results are fairly direct consequences of our Theorem 4 , simply because the sets $X$ and $Y$ described above partition the collection of maximal right ideals of $R$.

Finally, J. C. Robson in [15] introduced a localization as an inverse to the process of forming idealizers. Given a nonzero idempotent two-sided ideal $A$ in an $H N P$ ring $R$, the right order of $A$ is $O_{r}(A)=$ $\left\{x \in S^{0} R \mid A x \subseteq A\right\}$. If $R / A$ is a semisimple $\operatorname{ring}$, then $A$ is a semimaximal right ideal of $O_{r}(A)$ and $R$ is the idealizer of $A$ in $O_{r}(A)$ [15, Theorems 5.2,5.3]. Letting $X$ be the collection of those maximal left ideals of $R$ which do not contain $A$, we check that $R_{X}=O_{r}(A)$. Thus the inverse to taking right-hand idealizers is most naturally expressed as a left-hand localization, although in view of Theorem 5 it is also possible to express $O_{r}(A)$ as a right-hand localization.

4. Applications to splitting rings. We say that a ring $R$ is a (right) splitting ring provided that for every right $R$-module $A$, $Z(A)$ is a direct summand of $A$. In this section we use the localization techniques developed in $\S 2$ to answer the question of which $H N P$ rings are splitting rings.

Proposition 8. If $R$ is an HNP splitting ring, then every ring between $R$ and $S^{\circ} R$ is an HNP splitting ring.

Proof. Any ring $T$ between $R$ and $S^{0} R$ is an $H N P$ ring by Proposition 2. In view of Proposition 2(b), it follows immediately from [3, Proposition 1.9] that $T$ is also a splitting ring.

In order to describe the structure of $H N P$ splitting rings, we need the theory of idealizers developed by Robson in [15]. We now sketch the concepts involved, and refer to [15] for details. Given a right ideal $I$ in a ring $T$, the idealizer of $I$ in $T$ is the subring $S=\{t \in T \mid t I \subseteq I\}$. In general $S$ is unrelated to $T$ except for the case when $I$ is a semimaximal right ideal of $T$, i.e., a finite intersection of maximal right ideals of $T$. A subring $R$ of $T$ is an iterated idealizer from $T$ provided there is a chain of subrings 
$R_{0}=R \subseteq R_{1} \subseteq \cdots \subseteq R_{n}=T$ such that each $R_{i}$ is the idealizer of a semimaximal right ideal of $R_{i+1}$. There are theorems such as [15, Theorem 6.3] stating conditions under which an $H N P$ ring $R$ can be obtained as an iterated idealizer from a certain type of HNP ring $T$, and in these results the ring $T$ is always subring of the quotient ring $S^{0} R$.

THEOREM 9. An HNP ring $R$ is a splitting ring if and only if $R$ is an iterated idealizer from an HNP ring $T$ over which all singular right modules are injective.

(For a study of rings over which all singular right modules are injective, see [8, Chapter III].)

Proof. First assume that $R$ is such an iterated idealizer. Inasmuch as $T$ is clearly a splitting ring, it suffices to consider the case where $R$ is the idealizer of a semimaximal right ideal $M$ in an $H N P$ splitting ring $T$. If $M$ is an essential right ideal of $T$, then [9, Theorem 10] says that $R$ is a splitting ring. Otherwise $\operatorname{soc}\left(T_{T}\right) \neq 0$ and $T$ is a simple artinian ring. Consequently $M=e T$ for some idempotent $e \in T$, and then $R=e T+T(1-e)$. Inasmuch as $R$ is prime, the nilpotent two-sided ideal $e T(1-e)$ must be zero, and since $T$ is prime also we therefore have either $e=0$ or $e=1$. In either case $R=T$ and so $R$ is a splitting ring.

For the converse, we proceed via several lemmas. Our method is to show that at least one simple $R$-module is injective, and to use the localizations of $R$ to relate this fact to the other simple $R$-modules.

Lemma A. If $R$ is an HNP splitting ring, then at least one simple right $R$-module is injective.

Proof. We obviously may assume that $R$ is not semisimple.

Inasmuch as $R$ is right hereditary, every factor of the injective right $R$-module $Q=S^{0} R$ must be injective, hence it suffices to prove that $Q_{R}$ has a maximal submodule.

Since $R$ is not semisimple it must have a proper essential right ideal, and this essential right ideal must contain a nonzero-divisor $p$. Thus we obtain a properly descending chain $p R>p^{2} R>\cdots$ of essential right ideals of $R$. Noting that the module $R /\left(\cap p^{n} R\right)$ is not artinian, we see that $\cap p^{n} R \notin \mathscr{S}(R)$. We also define submodules $A_{1} \leqq A_{2} \leqq \cdots$ of $Q_{R}$ by setting $A_{1}=R$ and $A_{n+1} / A_{n}=\operatorname{soc}\left(Q / A_{n}\right)$ for all $n$. Inasmuch as every cyclic submodule of $Q / R$ has a composition series, we infer that $\bigcup A_{n}=Q$. 
Set $B_{n}=A_{n} / p^{n} R$ for each $n$, and let $x_{n}=\overline{1}$ in $B_{n}$. Since $R / p^{n} R$ has a composition series, it follows from the definition of the $A_{k}$ that every nonzero submodule of $B_{n}$ has a maximal submodule. Clearly the module $B=\prod_{n=1}^{\infty} B_{n}$ has the same property, hence we will be done if there exists a nonzero homomorphism of $Q$ into $B$. Inasmuch as $R$ is a splitting ring, the module $B / Z(B)$ is isomorphic to a direct summand of $B$, and thus it suffices to show that $\operatorname{Hom}_{R}(Q, B / Z(B)) \neq 0$.

The elements $x_{n} \in B_{n}$ are the components of an element $x \in B$ whose annihilator in $R$ is the right ideal $\bigcap p^{n} R$. Since $\bigcap p^{n} R \notin \mathscr{S}(R)$, we have $x \notin Z(B)$ and thus $f x \neq 0$, where $f: B \rightarrow B / Z(B)$ is the natural map.

For any given positive integer $k$ we can define an element $y_{k} \in B$ by setting $y_{k n}=x_{n}$ for $n=1, \cdots, k-1$ and $y_{k n}=0$ for $n \geqq k$. Noting that the modules $B_{n}$ are all singular, we see that in fact $y_{k} \in Z(B)$. Inasmuch as $A_{k} \leqq A_{n}$ for all $n \geqq k$, there are maps $g_{k n}: A_{k} \rightarrow B_{n}$ for each $n \geqq k$ such that $g_{k n} r=x_{n} r$ for all $r \in R$. For $n=1, \cdots, k-1$ we let $g_{k n}: A_{k} \rightarrow B_{n}$ denote the zero map, and then the maps $g_{k n}$ induce a map $g_{k}: A_{k} \rightarrow B$ such that $g_{k} r=\left(x-y_{k}\right) r$ for all $r \in R$.

We now have maps $f g_{k}: A_{k} \rightarrow B / Z(B)$ for each $k$ such that $f g_{k} r=(f x) r$ for all $r \in R$. Inasmuch as $B / Z(B)$ is nonsingular while the modules $A_{k} / R$ are all singular, we infer that $f g_{k}$ must be an extension of $f g_{j}$ whenever $j \leqq k$. This compatibility ensures that the maps $f g_{k}$ induce a map $h$ from $\bigcup A_{k}=Q$ into $B / Z(B)$, and we observe that $h 1=f x \neq 0$.

Lemma B. Let $R$ be an HNP splitting ring. If $S$ is any simple right $R$-module, then $\operatorname{Ext}_{R}^{1}(S, S)=0$.

Proof. Choose a maximal right ideal $M$ such that $R / M \cong S$, and recall from Theorem 3 that all simple right $R_{M}$-modules are isomorphic to $R_{M} / M R_{M}$. Inasmuch as $R_{M}$ is an $H N P$ splitting ring by Proposition 8 , Lemma A says that $R_{M} / M R_{M}$ is an injective $R_{M}$-module. Since $R_{M}$ is a flat left $R$-module, we now see from [12, Proposition 3, p. 131] that $R_{M} / M R_{M}$ is also injective as an $R$ module. Therefore, $R_{M} / M R_{M}$ is the injective hull of $f(R / M)$, where $f$ denotes the natural map $R / M \rightarrow R_{M} / M R_{M}$.

If $\operatorname{Ext}_{R}^{1}(S, S) \neq 0$, then there exists a nonsplit extension of $S$ by $S$, whence $E(S) / S$ contains a submodule isomorphic to $S$. But then $\left(R_{M} / M R_{M}\right) / f(R / M)$ contains a submodule isomorphic to $R / M$, which contradicts Lemma 6.

Lemma C. If $R$ is an HNP splitting ring, then any faithful simple right $R$-module $A$ is injective. 
Proof. Case $I . \quad R$ has exactly two simple right modules (up to isomorphism).

If we assume that $A$ is not injective, then according to Lemma A the other simple right $R$-module $B$ must be injective. Inasmuch as $\operatorname{Ext}_{R}^{1}(A, A)=0$ by Lemma $\mathrm{B}, E(A) / A$ has no submodules isomorphic to $A$, hence all its simple submodules are isomorphic to $B$. Now $E(A) / A$ has essential socle because it is singular, hence we infer from the injectivity of $B$ that $E(A) / A$ must be isomorphic to a direct sum of copies of $B$. This direct sum is nonzero because $A \neq E(A)$, and thus there exists an epimorphism of $E(A)$ onto $B$.

Choosing a maximal right ideal $M$ such that $R / M \cong A$, we infer as in Lemma $\mathrm{B}$ that $R_{M} / M R_{M} \cong E(A)$. Thus there exists an epimorphism of $R_{M} / M R_{M}$ onto $B$. By definition of $R_{M}$, the right $R$-module $R_{M} / R$ has no submodules isomorphic to $A$, hence it follows as with $E(A) / A$ above that $R_{M} / R$ must be isomorphic to a direct sum of copies of $B$. Therefore, there exists an epimorphism $f: T \rightarrow R_{M} / R$, where $T$ is a suitable direct sum of copies of $R_{M} / M R_{M}$.

Since $A$ is not injective, $R$ is not semisimple and thus $\operatorname{soc}\left(R_{R}\right)=0$. Thus $M$ must be essential in $R$ and so $A$ is singular. Then $R_{M} / M R_{M} \cong$ $E(A)$ is a singular $R$-module, hence $\operatorname{ker} f$ is singular too. Now $\operatorname{Ext}_{R}^{1}\left(R_{M}, \operatorname{ker} f\right)=0$ because $R$ is a splitting ring, whence the natural map $g: R_{M} \rightarrow R_{M} / R$ lifts to a map $h: R_{M} \rightarrow T$ such that $f h=g$. According to Proposition 2, $h$ is also an $R_{M}$-homomorphism, hence $K=\operatorname{ker} h$ is a right ideal of $R_{M}$.

Inasmuch as $T$ is a singular $R$-module whereas $R_{M}$ is a nonsingular $R$-module, we must have $K \neq 0$. Observing that $K \subseteq \operatorname{ker} g=R$, we see that $K$ is contained in the two-sided ideal $P=\left\{x \in R \mid x R_{M} \cong R\right\}$, and so $P \neq 0$. On the other hand, we have $R_{M} \neq R$ because these two rings have different numbers of simple right modules, and thus $P \neq R$.

The $R_{M}$-module $R_{M} / K$ is isomorphic to a submodule of the semisimple module $T$ and hence is semisimple itself. Observing that $P$ is a right ideal of $R_{M}$, we see that $R_{M} / P$ is a semisimple right $R_{M}$-module too. Now $R_{M} / P$ must be a direct sum of simple $R$-modules, each of which must be isomorphic to $R_{M} / M R_{M}$. Inasmuch as $R_{M} / M R_{M}$ has an essential $R$-submodule isomorphic to $R / M$ (and thus isomorphic to $A$ ), we infer that $R_{M} / P$ must have an essential $R$-submodule which is isomorphic to a direct sum of copies of $A$. Now $R / P \neq 0$, and thus $R / P$ must have an $R$-submodule isomorphic to $A$. Since $P$ is a two-sided ideal of $R$, it follows that $A P=0$, which contradicts the faithfulness of $A$.

Case II. General case. 
If $A$ is not injective, then $E(A) / A$ is a nonzero singular module and so contains a simple submodule $B$. Obviously $\operatorname{Ext}_{R}^{1}(B, A) \neq 0$, hence Lemma $\mathrm{B}$ says that $A \neq B$. Choosing maximal right ideals $M, N$ of $R$ such that $R / M \cong A$ and $R / N \cong B$, we see from Theorem 3 that $R_{M, N}$ has exactly two simple right modules (up to isomorphism). According to Proposition 8, $R_{M, N}$ is an $H N P$ splitting ring.

Inasmuch as $R_{M, N} / M R_{M, N}$ has an $R$-submodule isomorphic to $A$, it must be faithful as an $R$-module. Since $R$ is essential in $R_{M, N}$, it follows that $R_{M, N} / M R_{M, N}$ is also faithful as an $R_{M, N}$-module, hence we see from Case I that $R_{M, N} / M R_{M, N}$ is an injective $R_{M, N}$-module. As in Lemma $B$, we now infer that $R_{M, N} / M R_{M, N}$ is the injective hull of $f(R / M)$, where $f: R / M \rightarrow R_{M, N} / M R_{M, N}$ is the natural map. But then $\left(R_{M, N} / M R_{M, N}\right) / f(R / M)$ has a submodule isomorphic to $B$, which contradicts Lemma 6.

Lemma D. If $R$ is an HNP splitting ring, then $R$ has only finitely many maximal two-sided ideals, all of which are idempotent.

Proof. Assume to the contrary that there is an infinite sequence $M_{1}, M_{2}, \cdots$ of distinct maximal two-sided ideals of $R$. For each positive integer $n$, set $A_{n}=R / M_{n}$, which must be a singular right $R$-module because $M_{n} \neq 0$. Setting $x_{n}=\overline{1} \in A_{n}$, we note that $x_{n} \in A_{n} M_{k}$ for all $k \neq n$.

If $A=\prod_{n=1}^{\infty} A_{n}$, then $A=Z(A) \oplus B$ for some $B$. The elements $x_{n} \in A_{n}$ are the components of an element $x \in A$, and the annihilator $K=\{r \in R \mid x r=0\}$ is just the two-sided ideal $\cap M_{n}$. The ring $R / K$ has infinitely many maximal two-sided ideals and is therefore not artinian, whence $K=0$ and $x \notin Z(A)$. Thus $x=a+b$ for some $a \in Z(A)$ and some nonzero $b \in B$.

We must have $b_{k} \neq 0$ for some $k$. Define $z \in A$ by setting $z_{k}=x_{k}$ and $z_{n}=0$ for all $n \neq k$. Then $z \in Z(A)$ and $(x-z)_{n} \in A_{n} M_{k}$ for all $n$. Since $M_{k}$ is a finitely generated left ideal of $R$, we obtain $x-z \in A M_{k}$, from which it follows that $b \in B M_{k}$. But then $b_{k}=0$, which is a contradiction.

Therefore, $R$ has only finitely many maximal two-sided ideals. If $M$ is one of them, then either $M=0$ (in which case $M$ is automatically idempotent) or else $M \neq 0$ and $R / M$ is a simple artinian ring. In this case the ring $R / M^{2}$ has exactly one simple right module, say $S$, and $R / M$ and $M / M^{2}$ are each finite direct sums of copies of $S$. Inasmuch as $\operatorname{Ext}_{R}^{1}(S, S)=0$ by Lemma B, we obtain $\operatorname{Ext}_{R}^{1}(R / M$, $\left.M / M^{2}\right)=0$, from which it follows that $M / M^{2}=0$.

We now return to the proof of Theorem 9. If $R$ is an HNP splitting ring, then in view of Lemma $D$ we see from [15, Theorem 6.3] 
that $R$ is an iterated idealizer from a Dedekind prime ring T. Now $T$ is an $H N P$ splitting ring by Proposition 8 , and $T$ has no nontrivial idempotent two-sided ideals by [6, Theorem 1.2], hence it follows from Lemma $D$ that all maximal two-sided ideals of $T$ must be zero. Therefore, $T$ is a simple ring.

Now all simple right $T$-modules are faithful and hence injective by Lemma C. For any essential right ideal $I$ of $T, T / I$ has a composition series and so must now be semisimple. According to [8, Proposition 3.1], it follows that all singular right $T$-modules are injective.

According to [3, Theorem 2.1], a commutative ring $R$ is a splitting ring if and only if all singular $R$-modules are injective, whereas Theorem 9 allows a noncommutative $H N P$ splitting ring to be a finite number of idealizations aways from a ring over which all singular modules are injective. We now construct an example to show that there is a real distinction between these two situations. First let $T$ be the ring constructed in [4, Theorem 1.4]: $T$ is a principal right and left ideal domain, $T$ is a simple ring but not a division ring, and all simple right $T$-modules are injective. As shown in $[8, \mathrm{pp} .54,55]$, all singular right $T$-modules are injective as well. We now choose a maximal right ideal $M$ of $T$, and let $R$ be the idealizer of $M$ in $T$. Inasmuch as $T$ is not a division ring, $M$ is essential in $T$ and in particular $M \neq 0$. Then $T M=T$ because $T$ is simple, so [15, Theorem 5.3] shows that $R$ is an HNP ring and Theorem 9 says that $R$ is a splitting ring. According to [15, Theorem 1.3], the right $R$-module $T / M$ has a unique composition series given by $T / M>R / M>0$, hence $R / M$ is a singular right $R$-module which is not injective.

THEOREM 10. Let $R$ be an HNP ring. Then $R$ is a splitting ring if and only if

(a) $R$ contains a minimal (nonzero) two-sided ideal.

(b) All faithful simple right $R$-modules are injective.

Proof. If $R$ is a splitting ring, then we have (b) by Lemma C. In case $R$ is simple, then $R$ itself is a minimal two-sided ideal, hence in proving (a) we need only consider the case when $R$ is not simple.

According to [11, Theorem 2.24], $R$ is the intersection of two subrings $S$ and $T$ of $S^{0} R$ such that $S$ is a bounded HNP ring, while $T$ is an $H N P$ ring with no proper invertible two-sided ideals. We claim that $T=R$, and to show this it suffices to prove that $S=S^{0} R$, i.e., that $S$ is a simple artinian ring.

Inasmuch as $S$ is a splitting ring by Proposition 8, Lemma A 
says that $S$ has a maximal right ideal $M$ such that $S / M$ is injective. The injectivity of $S / M$ implies that $(S / M) p=S / M$ for all non-zerodivisors $p \in S$, from which we infer that $(S / M) I=S / M$ for all nonzero two-sided ideals $I$ of $S$. Therefore, $M$ cannot contain any nonzero two-sided ideals of $S$. However, $S$ is bounded, hence we infer from this that $M$ is not essential in $S$. Thus $\operatorname{soc}\left(S_{S}\right) \neq 0$ and so $S$ is indeed a simple artinian ring.

Now $T=R$ as claimed, hence $R$ has no proper invertible twosided ideals. According to Lemma D, $R$ has only finitely many maximal two-sided ideals, say $M_{1}, \cdots, M_{n}$, and these ideals are all nonzero because $R$ is not a simple ring. If $M=M_{1} \cap \cdots \cap M_{n}$, then [6, Proposition 4.3] shows that $M^{n}$ is idempotent, and we observe that $M^{n} \neq 0$. Now for any nonzero two-sided ideal $H$ of $R$, the factor ring $R / H$ is artinian, hence its radical $N / H$ is nilpotent and is an intersection of maximal two-sided ideals. Then $M \cong N$, so the nilpotence of $N / H$ and the idempotence of $M^{n}$ combine to show that $M^{n} \subseteq H$. Therefore, $M^{n}$ is a minimal (in fact minimum) twosided ideal of $R$.

Conversely, assuming that (a) and (b) hold, we must show that $\operatorname{Ext}_{R}^{1}(A, C)=0$ for any nonsingular $A_{R}$ and any singular $C_{R}$. Letting $H$ denote a minimal two-sided ideal of $R$, we infer from the fact that $R$ is prime that $H=H^{2}$ and that $H$ is contained in all nonzero two-sided ideals of $R$. Thus all unfaithful $R$-modules are annihilated by $H$.

If $C^{\prime}=\{x \in C \mid x H=0\}$, then from $H=H^{2}$ we see that no nonzero elements of $C / C^{\prime}$ are annihilated by $H$. Therefore, all nonzero submodules of $C / C^{\prime}$ are faithful, hence we see from (b) that all simple submodules of $C / C^{\prime}$ are injective. Now $C / C^{\prime}$ has essential socle because it is singular, hence we infer that $C / C^{\prime}$ is injective. Therefore $\operatorname{Ext}_{R}^{1}\left(A, C / C^{\prime}\right)=0$ and so it suffices to show that $\operatorname{Ext}_{R}^{1}\left(A, C^{\prime}\right)=0$.

As observed above, all nonsingular right $R$-modules are flat, hence the right-hand version of [2, Proposition 4.1.3, p. 118] says that $\operatorname{Ext}_{R}^{1}\left(A, C^{\prime}\right) \cong \operatorname{Ext}_{R / H}^{1}\left(A / A H, C^{\prime}\right)$. Now $R / H$ is an artinian ring because $H \neq 0$, and $A / A H$ is a flat $(R / H)$-module because $A_{R}$ is flat, whence $(A / A H)_{R / H}$ must be projective. Therefore, $\operatorname{Ext}_{R / H}^{1}\left(A / A H, C^{\prime}\right)=0$ and so $\operatorname{Ext}_{R}^{1}\left(A, C^{\prime}\right)=0$.

Note added in proof. Many of the results in $\$ 2$ have also been proved (independently) for the case of a Dedekind prime ring by $\mathrm{H}$. Marubayashi. 


\section{REFERENCES}

1. K. Asano, Arithmetik in Schiefringen I, Osaka Math. J., 1 (1949), 98-134.

2. H. Cartan and S. Eilenberg, Homological Algebra, Princeton University Press, Princeton, 1956.

3. V. C. Cateforis and F. L. Sandomierski, The singular submodule splits off, J. Algebra, 10 (1968), 149-165.

4. J. H. Cozzens, Homological properties of the ring of differential polynomials, Bull. Amer. Math. Soc., 76 (1970), 75-79.

5. D. Eisenbud and J. C. Robson, Modules over Dedekind prime rings, J. Algebra, 16 (1970), 67-85.

6. D. Eisenbud and J. C. Robson, Hereditary noetherian prime rings, J. Algebra, 16 (1970), 86-104.

7. O. Goldman, Rings and modules of quotients, J. Algebra, 13 (1969), 10-47.

8. K. R. Goodearl, Singular torsion and the splitting properties, Amer. Math. Soc. Memoirs, No. 124 (1972).

9. - Idealizers and nonsingular rings, Pacific J. Math., 48 (1973), 395-402.

10. J. Kuzmanovich, Localizations of Dedekind prime rings, J. Algebra, 21 (1972), 378-393.

11. - Localizations of HNP rings, Trans. Amer. Math. Soc., 173 (1972), 137157.

12. J. Lambek, Lectures on Rings and Modules, Blaisdell, Waltham, Mass., 1966.

13. T. H. Lenagan, Bounded Asano orders are hereditary, Bull. London Math. Soc., 3 (1971), 67-69.

14. J. C. Robson, Noncommutative Dedekind rings, J. Algebra, 9 (1968), 249-265.

15. - Idealizers and hereditary noetherian prime rings, J. Algebra, 22 (1972), $45-81$.

16. F. L. Sandomierski, Nonsingular rings, Proc. Amer. Math. Soc., 19 (1968), 225230 .

17. L. Silver, Noncommutative localizations and applications, J. Algebra, 7 (1967), 44-76.

18. D. C. Webber, Ideals and modules of simple noetherian hereditary rings, J. Algebra, 16 (1970), 239-242.

Received July 5, 1973 and in revised form October 10, 1973.

UNIVERSITY OF UTAH 



\section{PACIFIC JOURNAL OF MATHEMATICS}

\section{EDITORS}

RICHARD ARENS (Managing Editor)

University of California

Los Angeles, California 90024

R. A. Beaumont

University of Washington

Seattle, Washington 98105
J. DugundJI

Department of Mathematics

University of Southern California

Los Angeles, California 90007

D. Gilbarg and J. Milgram

Stanford University

Stanford, California 94305

\section{ASSOCIATE EDITORS}

E. F. BECKENBACH

B. H. NeUMANN

F. WOLF

K. YosHIDA

\section{SUPPORTING INSTITUTIONS}

UNIVERSITY OF BRITISH COLUMBIA

CALIFORNIA INSTITUTE OF TECHNOLOGY

UNIVERSITY OF CALIFORNIA

MONTANA STATE UNIVERSITY

UNIVERSITY OF NEVADA

NEW MEXICO STATE UNIVERSITY

OREGON STATE UNIVERSITY

UNIVERSITY OF OREGON

OSAKA UNIVERSITY
UNIVERSITY OF SOUTHERN CALIFORNIA

STANFORD UNIVERSITY

UNIVERSITY OF TOKYO

UNIVERSITY OF UTAH

WASHINGTON STATE UNIVERSITY

UNIVERSITY OF WASHINGTON

$\stackrel{*}{*} \stackrel{*}{*} \stackrel{*}{*}$ AMERICAN MATHEMATICAL SOCIETY




\section{Pacific Journal of Mathematics}

\section{Vol. 53, No. $1 \quad$ March, 1974}

Martin Bartelt, Strongly unique best approximates to a function on a set, and a finite

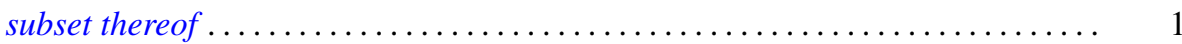

S. J. Bernau, Theorems of Korovkin type for $L_{p}$-spaces $\ldots \ldots \ldots \ldots \ldots \ldots \ldots \ldots \ldots$

S. J. Bernau and Howard E. Lacey, The range of a contractive projection on an

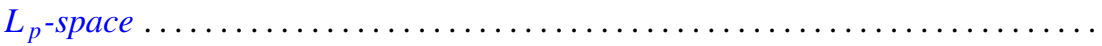

Marilyn Breen, Decomposition theorems for 3-convex subsets of the plane ......... Ronald Elroy Bruck, Jr., A common fixed point theorem for a commuting family of

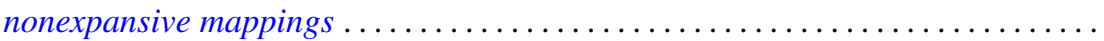

Aiden A. Bruen and J. C. Fisher, Blocking sets and complete $k$-arcs . . . . . . . 73

R. Creighton Buck, Approximation properties of vector valued functions . ......... 85

Mary Rodriguez Embry and Marvin Rosenblum, Spectra, tensor products, and

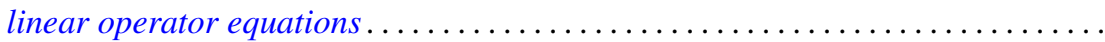

Edward William Formanek, Maximal quotient rings of group rings . . . . . . . . . 109

Barry J. Gardner, Some aspects of T-nilpotence . . . . . . . . . . . . . . . 117

Juan A. Gatica and William A. Kirk, A fixed point theorem for $k$-set-contractions

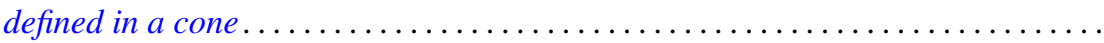

Kenneth R. Goodearl, Localization and splitting in hereditary noetherian prime

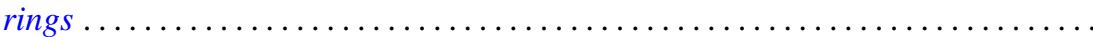

James Victor Herod, Generators for evolution systems with quasi continuous

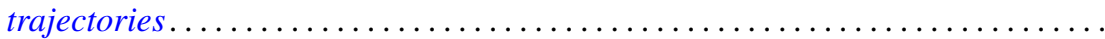

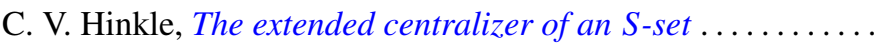

I. Martin (Irving) Isaacs, Lifting Brauer characters of p-solvable groups . . .

Bruce R. Johnson, Generalized Lerch zeta function ...........

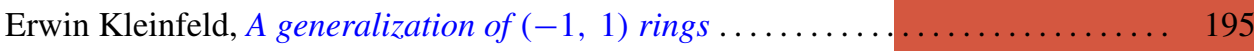

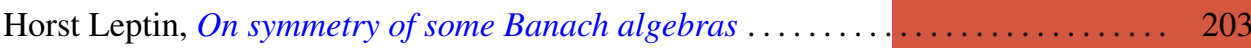

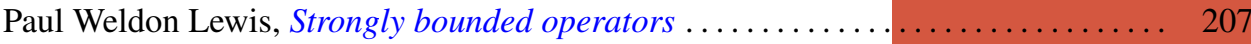

Arthur Larry Lieberman, Spectral distribution of the sum of self-adjoint

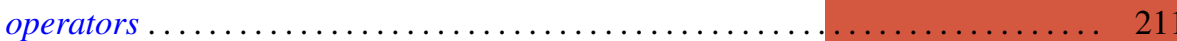

I. J. Maddox and Michael A. L. Willey, Continuous operators on paranormed spaces and matrix transformations

James Dolan Reid, On rings on groups ........................... 229

Richard Miles Schori and James Edward West, Hyperspaces of graphs are Hilbert cubes.

William H. Specht, A factorization theorem for p-constrained groups ...

Robert L Thele, Iterative techniques for approximation of fixed points of certain nonlinear mappings in Banach spaces ...............

Tim Eden Traynor, An elementary proof of the lifting theorem

Charles Irvin Vinsonhaler and William Jennings Wickless, Completely decomposable groups which admit only nilpotent multiplications .

Raymond O’Neil Wells, Jr, Comparison of de Rham and Dolbeault cohomology for

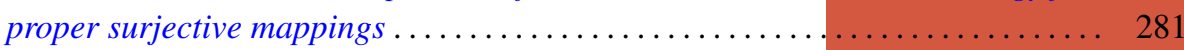

David Lee Wright, The non-minimality of induced central representations . . . . . 301 